\title{
Potentiometric aptasensing of small molecules based on surface charge change
}

\author{
Enguang $\mathrm{LV}^{\mathrm{a}, \mathrm{b}}$, Jiawang Ding ${ }^{\mathrm{a}, *}$, Wei Qin ${ }^{\mathrm{a}, *}$ \\ ${ }^{a}$ Key Laboratory of Coastal Environmental Processes and Ecological Remediation, Yantai Institute of Coastal Zone Research (YIC), Chinese Academy of \\ Sciences (CAS), Shandong Provincial Key Laboratory of Coastal Environmental Processes, YICCAS, Yantai, Shandong 264003, PR China \\ ${ }^{\mathrm{b}}$ University of the Chinese Academy of Sciences, Beijing 100049, PR China
}

\section{A R T I C L E I N F O}

\section{Article history:}

Received 11 August 2017

Received in revised form

23 November 2017

Accepted 11 December 2017

Available online 15 December 2017

\section{Keywords:}

Aptasensor

Bisphenol A

Potentiometry

Surface charge

Solid state

\begin{abstract}
A B S T R A C T
Solid-state potentiometric sensors based on surface charge change are well-established tools for measuring ions and biological species. However, their use for detection of small molecules with high sensitivity and good selectivity is still elusive. In this work, a novel potentiometric aptasensing platform for small molecules is presented, using bisphenol A (BPA) as a model. The proposed sensor can be prepared by layer-by-layer assembling of carboxylated multiwall carbon nanotubes, poly(diallyldimethylammonium chloride) (polycation), and aptamer (polyanion) on the electrode surface. The presence of BPA induces the conformational change and detachment of the aptamer at the surface of the modified electrode, which leads to a variation of the surface charge (negative to positive) and therefore a potential change. The introduction of polyions can cause substantial charge change on the electrode surface, thus improving the sensitivity of the sensor. The morphology and electron-transfer properties of the electrode have been characterized. Under optimum conditions, the present sensor shows a stable response to BPA in the concentration range from $3.2 \times 10^{-8}$ to $1.0 \times 10^{-6} \mathrm{M}$ with a detection limit of $1.0 \times 10^{-8} \mathrm{M}$. The proposed methodology can be used for sensitive potentiometric sensing of other small molecules involved in aptamer/target binding events.
\end{abstract}

(c) 2017 Elsevier B.V. All rights reserved.

\section{Introduction}

Potentiometric sensors based on solvent polymeric membrane ion-selective electrodes (ISEs) were developed in the 1970s [1]. In recent years, they have been improved in terms of sensitivity, selectivity, reversibility, and reliability [2-4]. With the introduction of various synthetic receptors, new materials, and deeper theoretical models [5-8], potentiometry based on solvent polymeric membrane ISEs has become a well-established tool for ion analysis. In parallel, researchers have designed solid-state potentiometric sensors, which are tailored with recognition elements on the surfaces of the electrodes. In these solid-state sensors, the target-induced adsorption, desorption, or uptake of ions or charged molecules on the surfaces of electrodes can change the surface potentials of the electrodes [9-12].

Early researches on solid-state sensors focused mainly on the detection of ions such as $\mathrm{Cu}^{2+}$ and $\mathrm{Pb}^{2+}$ by using the precipitatebased electrodes $[10,13]$. The introduction of nanomaterials and

\footnotetext{
* Corresponding authors.

E-mail addresses: jwding@yic.ac.cn (J. Ding), wqin@yic.ac.cn (W. Qin).
}

novel recognition molecules was a key turning point in this field. In 2012, Michalska et al. [14] developed a potentiometric sensor based on the dithizone modified gold nanoparticle film for the determination of copper ions. In this method, the cations diffuse into the gold nanoparticle layer and interact with dithizone to produce the surface charge change. Similarly, a solid-state potentiometric sensor with improved selectivity for copper ions was designed using an ion-selective gold-thiol film [15].

Solid-state potentiometric sensors for macromolecules and whole cells have also been developed. In 1975, Janata developed an immunoelectrode based on surface recognition [16]. The target antigen with net electrical charges could induce a charge change on the surface, along with a subsequent potential change. However, the low efficiency of the immobilization procedures restrict its wide applications. Subsequently, research in this area encountered a bottleneck until the introduction of novel materials and the optimization of the modification processes. In 2009, Rius et al. [17] developed a potentiometric aptasensor using aptamer functionalized single-walled carbon nanotubes as a transducing material for immediate detection of living bacteria. Although the sensor could detect bacteria rapidly, the potential changes are small, even with the introduction of dual aptamers [18]. So far, none solid-state 
potentiometric sensors have been developed for sensitive detection of small molecules.

In this paper, a novel potentiometric aptasensing platform for small molecules based on surface charge change is proposed. Bisphenol A (BPA), which is an endocrine disrupter, was selected as a model. An aptamer with excellent selectivity and high binding affinity to BPA is used as a recognition element [19]. In recent years, a number of aptasensors for BPA based on optical, electrochemical, and mass-sensitive transductions have been developed [20,21]. Among these methods, electrochemical aptasensors have been received considerable attention due to their advantages of rapid response, ease of use, and low cost. As one kind of simple, field-portable, and widespread electrochemical methodology, potentiometric sensors have been adopted as promising transducers for aptasensing. In our previous research, a potentiometric aptasensing strategy based on DNA nanostructures has been designed for the detection of BPA with high sensitivity [22]. However, our previous system for BPA needs additional signal reporter of protamine, thus adding more complexity. Herein, a simple and direct potentiometric aptasensing platform is designed via a layer-by-layer technique. For the present work, the charged materials, carboxylated multiwall carbon nanotubes (CNTs), poly(diallyldimethylammonium chloride) (PDDA, polycation), and the aptamer (polyanion), are immobilized layer-by-layer on the electrode surface. The target-binding induced release of the aptamer from the surface of the electrodes causes a charge distribution change and subsequently a potential change. It is anticipated that the introduction of an aptamer for the specific target allows the sensing methodology with high generality and good selectivity.

\section{Experimental section}

\subsection{Materials}

An aptamer with a dissociation constant of $8.3 \mathrm{nM}$ toward BPA was used [19]. The sequence was: 5'-CCG GTG GGT GGT CAG GTG GGA TAG CGT TCC GCG TAT GGC CCA GCG CAT CAC GGG TTC GCA CCA-3'. More information of the materials is available in the Supporting Information.

\subsection{Electrode preparation}

As illustrated in Scheme 1, chemically modified glass carbon electrodes (GC/CNTs/PDDA/aptamer) were fabricated using the layer-by-layer technique. The detailed procedures are shown in the Supporting Information.

\subsection{Electrochemical measurements}

Potentiometric measurements were performed using a doublejunction $\mathrm{Ag} / \mathrm{AgCl}(3.0 \mathrm{M} \mathrm{KCl})$ reference electrode with $1.0 \mathrm{M} \mathrm{LiOAc}$ as a salt bridge electrolyte in the galvanic cell as follows: $\mathrm{Ag} / \mathrm{AgCl} / 3.0 \mathrm{M} \mathrm{KCl} / 1.0 \mathrm{M} \mathrm{LiOAc/binding} \mathrm{buffer/modified} \mathrm{electrode.}$ The binding buffer was Tris-buffered saline solution (50 mM Tris base, $100 \mathrm{mM} \mathrm{NaCl}, 25 \mathrm{mM} \mathrm{KCl}, 10 \mathrm{mM} \mathrm{MgCl}_{2}$, pH 7.4). The electromotive force (EMF) measurements were carried out using a $\mathrm{CHI}$ 760D electrochemical workstation (Shanghai Chenhua Apparatus Corporation, China). In this work, the open circuit potential (OCP) of the proposed sensor recorded in the binding buffer was used as the baseline. Then, $20 \mu \mathrm{L}$ of BPA at different concentrations was added on the electrode surface for incubation. After incubation for $15 \mathrm{~min}$ and washing with binding buffer, the potential response was recorded in the binding buffer. The potential change between the baseline and the potential recorded at $100 \mathrm{~s}$ was used for quantification of BPA.
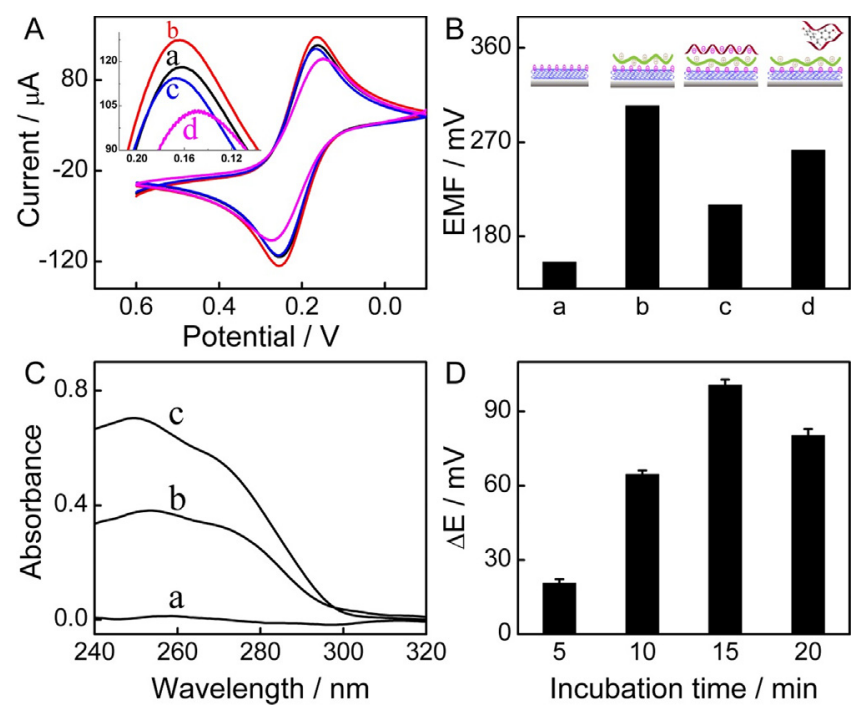

Fig. 1. (A) Cyclic voltammograms recorded in $0.1 \mathrm{M} \mathrm{KCl}$ containing $5 \mathrm{mM}$ $\mathrm{Fe}(\mathrm{CN})_{6}{ }^{4-/ 3-}$, for the electrodes: (a) GC, (b) GC/CNTs, (c) GC/CNTs/PDDA, and (d) GC/CNTs/PDDA/aptamer. (B) Open circuit potentials of (a) GC/CNTs, (b) GC/CNTs/PDDA, (c) GC/CNTs/PDDA/aptamer, and (d) GC/CNTs/PDDA/aptamer after incubation with $10^{-7} \mathrm{M}$ BPA for $15 \mathrm{~min}$. Potential responses of the modified electrodes were recorded for $100 \mathrm{~s}$. (C) UV spectra of (a) the incubation solution alone after incubation for $15 \mathrm{~min}$, and (b) the incubation solution in the presence of $10^{-5}$ BPA after incubations for 10 , and (c) $15 \mathrm{~min}$ with the GC/CNTs/PDDA/aptamer electrode. $5 \mu \mathrm{L}$ of $10^{-5} \mathrm{M}$ aptamer was used to prepare the electrode. (D) Potentiometric responses of the electrodes to $10^{-6} \mathrm{M} \mathrm{BPA}$ with different incubation times. Error bars represent one standard deviation for three measurements.

\section{Results and discussion}

As illustrated in Scheme 1, CNTs, PDDA, and the aptamer were successively modified on the electrode via electrostatic and $\pi-\pi$ interactions, thus forming an electric double layer at the electrode/solution interface. The presence of BPA could induce the conformational change and further detachment of the aptamer from the surface of the modified electrode. This causes the charge distribution change of the electric double layer and therefore induces a potential change. Indeed, previous reports demonstrated that an increase of any excess negative or positive charges on the surface of the electrode can lead to a potential response [16,23]. More importantly, polyions with large amounts of charges assembled on the surface could induce much larger surface charge changes for improving the sensitivity of the potentiometric sensors.

To characterize the fabrication process of the layer-by-layer assembly, cyclic voltammetry (CV) was performed (Fig. 1A). Compared with the bare glass carbon electrode (GC) (Fig. 1A, curve a), the electrode modified with CNTs exhibits better conductivity (Fig. 1A, curve b). However, PDDA and the aptamer could block the electron transfer and induce lower peak currents (Fig. 1A, curve $\mathrm{C}$ and d). Field-emission scanning electron microscopy pictures also indicate the layer-by-layer modification process (Fig. S1). Moreover, the charge distribution or density on the electrode surface will change during the process of the layer-by-layer assembly. Therefore, the open circuit potentials of the modified electrode were measured (Fig. 1B). Compared with GC/CNTs (Fig. 1B, column a), the modification of PDDA with a large positive charge changes the charge distribution on the electrode surface from negative to positive, which induces a large increase of the potential change (Fig. 1B, column b). Furthermore, the adsorption of aptamer leads to a decrease of the OCP (Fig. 1B, column c). These results demonstrate that the electrode was successfully prepared by the layer-by-layer assembly. 


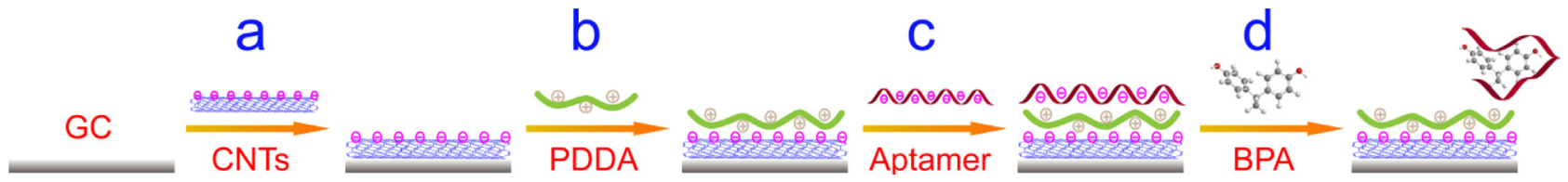

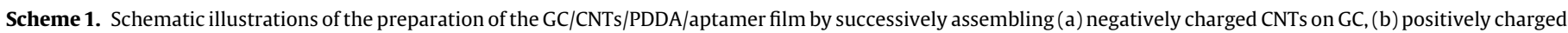
PDDA, and (c) negatively charged aptamer, and (d) the interaction with the target.

According to Fig. 1B (column d), the incubation of the modified electrode with the target molecules induces the recovery of the OCP, which is probably due to the detachment or release of the aptamer into the sample solution. The release of the aptamer from the electrode was confirmed by using UV spectroscopy. In contrast to the incubation solution without BPA (Fig. 1C, curve a), the incubation solution with BPA causes an obvious UV absorption (Fig. 1C, curve b). Moreover, the amount of released aptamer can be increased by increasing the incubation time (Fig. 1C, curve c).

A similar phenomenon can also be observed when potentiometric measurements with different incubation times were carried out. During the incubation, a conformational change of the aptamer occurred that weakened the interactions between PDDA and the aptamer. The conjugate of the aptamer and BPA dropped away when the electrodes were rinsed or placed in the stirring solution. The decrease of the aptamer on the electrode surface induced the surface charge change of the working electrode, thus changing the potential of the electrode. Furthermore, the higher binding constant of BPA and the aptamer made the separation between the aptamer and PDDA easier [24]. As shown in Fig. 1D, the response of the modified electrode increases with the incubation time for up to $15 \mathrm{~min}$, and then decreases gradually, which might be duo to the dissolution of PDDA. The incubation time of $15 \mathrm{~min}$ was selected for further experiments.

BPA at different concentrations could lead to the detachments of aptamer to variable extents. The potential responses to BPA in the concentration range of $1.0 \times 10^{-9}$ to $1.0 \times 10^{-6} \mathrm{M}$ are shown in Fig. 2A. Under the optimal experimental conditions, the sensor exhibits a linear response range from $3.2 \times 10^{-8}$ to $1.0 \times 10^{-6} \mathrm{M}$ with a slope of $52.7 \mathrm{mV} / \mathrm{dec}$. The detection limit was calculated to be $1.0 \times 10^{-8} \mathrm{M}(3 \sigma)$. In our previous research, a lower detection limit of 80 pM for BPA could be obtained, which is probably due to the multiple target-binding sites (aptamer) on each of the long concatamer [22]. Compared with our previous aptasensing platform that couples to a signal amplification strategy based on DNA assembly, the present sensor is simple and easy to use. More importantly, the proposed sensor exhibits better sensitivity than that of the existing potentiometric solid-state sensors based on surface charge change, which were designed for proteins and whole cells [17,25-27].

Without the polycation, the aptamer also could be modified on the electrode by utilizing $\pi-\pi$ stacking interactions between the nucleic acid bases and the carbon nanotubes' walls [28]. The fabricated electrode (GC/CNTs/aptamer) was tested for the detection of BPA. Likewise, the target/aptamer recognition could release the aptamer from the CNTs layer, inducing the change of surface potential. Fig. 2 shows that the sensor indeed responds to BPA at different concentrations (Fig. 2B, a). However, the sensor shows a poor performance in sensitivity (Fig. 2B, b).

Control experiments reveal that no significant potential change was observed for the control DNA (Fig. 2C). More importantly, the presence of other structurally similar molecules (bisphenol B, biphenyl, OH-biphenyl and 2-OH-biphenyl) could not lead to an obvious potential change (Fig. 2D), which confirms the specific interaction between BPA and the aptamer.

To investigate the regeneration of the proposed sensor, 5 regeneration cycles were tested. As shown in Fig. S3, a decrease of ca $20 \%$
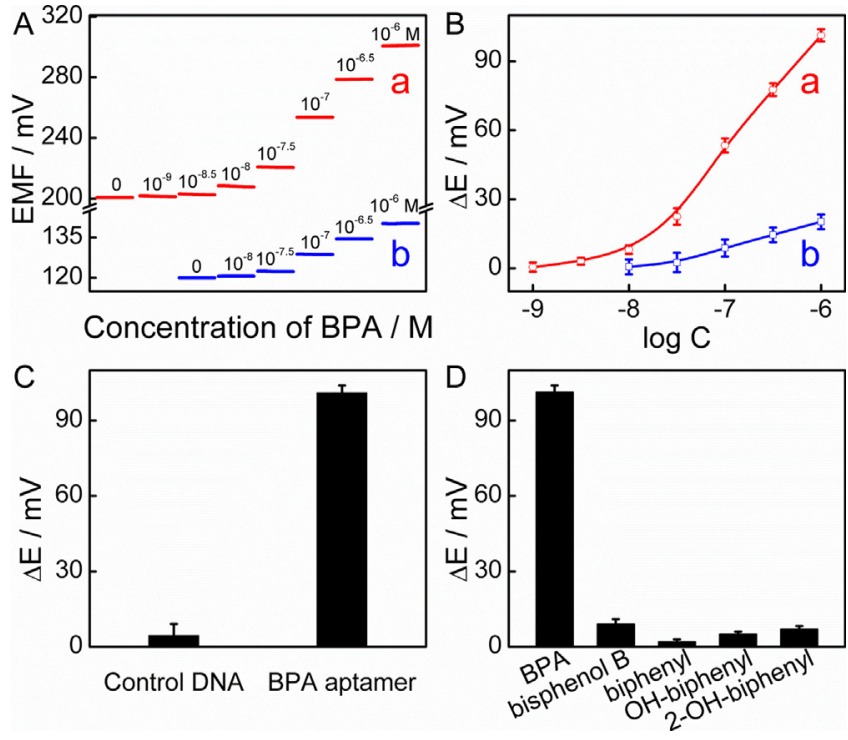

Fig. 2. (A) Potential responses of the electrodes after incubation in binding buffer with BPA of $10^{-9}, 10^{-8.5}, 10^{-8}, 10^{-7.5}, 10^{-7}, 10^{-6.5}$, and $10^{-6} \mathrm{M}$ with (a) the GC/CNTs/PDDA/aptamer electrode and (b) the GC/CNTs/aptamer electrode. Potential responses of the modified electrodes were recorded for $100 \mathrm{~s}$. (B) Calibration curves for BPA with (a) the GC/CNTs/PDDA/aptamer electrode and (b) the GC/CNTs/aptamer electrode, respectively. (C) The potential changes of the control DNA and BPA aptamer modified electrode in the presence of $10^{-6} \mathrm{M}$ BPA. (D) Potential responses of the electrodes to BPA and other structurally similar molecules. The concentrations of BPA and its analogues were $10^{-6} \mathrm{M}$. Error bars represent one standard deviation for three measurements.

of the potentiometric response was observed after 3 regeneration cycles, which is probably due to the release of PDDA from the membrane into the aqueous sample solution. Experiments revealed that no obvious loss of response was observed after dry storage of the modified electrode at $4{ }^{\circ} \mathrm{C}$ for 7 days (see Supporting Information, Fig. S4). The deterioration of the sensor response could be attributed to the dissolution of PDDA.

\section{Conclusions}

In summary, a potentiometric aptasensing platform for small molecules based on surface charge change has been demonstrated. Polyions with large amounts of charges can be introduced and modified on the electrode for improving sensitivity. The target induced detachment of the aptamer leads to the surface charge change from negative to positive, which can amplify the potential response. Our proposed approach significantly improves the sensitivity and can be extended to determine other analytes that can interact with their aptamers, such as bacteria, proteins, and metal ions. Further applications of the present sensing configuration are currently in progress in our laboratory.

\section{Acknowledgments}

This work was supported by the National Natural Science Foundation of China $(21575158,21475148)$, the National Key Research 
and Development Program of China (2016YFC1400700), the Instrument Developing Project of the Chinese Academy of Sciences (Y728021021), the CAS Youth Innovation Promotion Association (2014190), and the Taishan Scholar Program of Shandong Province (TS20081159).

\section{Appendix A. Supplementary data}

Supplementary data associated with this article can be found, in the online version, at https://doi.org/10.1016/j.snb.2017.12.067.

\section{References}

[1] E. Bakker, P. Bühlmann, E. Pretsch, Carrier-based ion-selective electrodes and bulk optodes. 1. General characteristics, Chem. Rev. 97 (1997) 3083-3132.

[2] E. Bakker, E. Pretsch, Modern potentiometry, Angew. Chem. Int. Ed. 46 (2007) 5660-5668.

[3] J. Bobacka, A. Ivaska, A. Lewenstam, Potentiometric ion sensors, Chem. Rev. 108 (2008) 329-351.

[4] E. Bakker, Electroanalysis with membrane electrodes and liquid-liquid interfaces, Anal. Chem. 88 (2016) 395-413.

[5] J.B. Hu, A. Stein, P. Bühlmann, Rational design of all-solid-state ion-selective electrodes and reference electrodes, TrAC-Trend Anal. Chem. 76 (2016) $102-114$.

[6] J.W. Ding, Y. Chen, X.W. Wang, W. Qin, Label-free and substrate-free potentiometric aptasensing using polycation-sensitive membrane electrodes, Anal. Chem. 84 (2012) 2055-2061.

[7] R.N. Liang, D.A. Song, R.M. Zhang, W. Qin, Potentiometric sensing of neutral species based on a uniform-sized molecularly imprinted polymer as a receptor, Angew. Chem. Int. Ed. 49 (2010) 2556-2559.

[8] T. Sokalski, T. Zwickl, E. Bakker, E. Pretsch, Lowering the detection limit of solvent polymeric ion-selective electrodes. 1. Modeling the influence of steady-state ion fluxes, Anal. Chem. 71 (1999) 1204-1209.

[9] V. Agarwala, M.C. Chattopadhyaya, A heterogeneous precipitate based Mn(II) coated wire ion-selective electrode, Anal. Lett. 22 (1989) 1451-1457.

[10] E.G. Harsányi, K. Tóth, E. Pungor, The adsorption of copper ions on the surface of copper(II) sulfide precipitate-based ion-selective electrodes, Anal. Chim. Acta 152 (1983) 163-171

[11] E.G. Harsányi, K. Tóth, E. Pungor, Y. Umezawa, S. Fujiwara, Study of the potential response of solid-state chloride electrodes at low concentration ranges, Talanta 31 (1984) 579-584.

[12] Y. Tani, H. Fun, Y. Umezawa, A cation selective electrode based on copper(II) and nickel(II) hexacyanoferrates: dual response mechanisms selective uptake or adsorption of analyte cations, Electrochim. Acta 43 (1998) 3431-3441.

[13] E. Pungor, K. Tóth, Precipitate-based ion-selective electrodes, Pure Appl. Chem. 34 (2009) 105-137.

[14] E. Woźnica, M.M. Wójcik, M. Wojciechowski, J. Mieczkowski, E. Bulska, K. Maksymiuk, A. Michalska, Dithizone modified gold nanoparticles films for potentiometric sensing, Anal. Chem. 84 (2012) 4437-4442.

[15] M. Li, H. Zhou, L. Shi, D.W. Li, Y.T. Long, Ion-selective gold-thiol film on integrated screen-printed electrodes for analysis of Cu(II) ions, Analyst 139 (2014) 643-648.

[16] J. Janata, Immunoelectrode, J. Am. Chem. Soc. 97 (1975) 2914-2916.
[17] G.A. Zelada-Guillén, J. Riu, A. Düzgün, F.X. Rius, Immediate detection of living bacteria at ultralow concentrations using a carbon nanotube based potentiometric aptasensor, Angew. Chem. Int. Ed. 48 (2009) 7334-7337.

[18] T. Goda, D. Higashi, A. Matsumoto, T. Hoshi, T. Sawaguchi, Y. Miyahara, Dual aptamer-immobilized surfaces for improved affinity through multiple target binding in potentiometric thrombin biosensing, Biosens. Bioelectron. 73 (2015) 174-180

[19] M. Jo, J.Y. Ahn, J. Lee, S. Lee, S.W. Hong, J.W. Yoo, J. Kang, P. Dua, D.K. Lee, S. Hong, S. Kim, Development of single-stranded DNA aptamers for specific bisphenol A detection, Oligonucleotides 21 (2011) 85-91.

[20] K.V. Ragavan, N.K. Rastogi, M.S. Thakur, Sensors and biosensors for analysis of bisphenol-A, TrAC-Trend Anal. Chem. 52 (2013) 248-260.

[21] H. Mirzajani, C. Cheng, J. Wu, J. Chen, S. Eda, E. Najafi Aghdam, H. Badri Ghavifekr, A highly sensitive and specific capacitive aptasensor for rapid and label-free trace analysis of bisphenol A (BPA) in canned foods, Biosens. Bioelectron. 89 (2017) 1059-1067.

[22] J.W. Ding, Y. Gu, F. Li, H.X. Zhang, W. Qin, DNA nanostructure-based magnetic beads for potentiometric aptasensing, Anal. Chem. 87 (2015) 6465-6469.

[23] R. Resnick, D. Halliday, Physics, 3rd ed., John Wiley \& Sons Inc., 1978.

[24] Y. Sultan, R. Walsh, C. Monreal, M.C. DeRosa, Preparation of functional aptamer films using layer-by-layer self-assembly, Biomacromolecules 10 (2009) 1149-1154.

[25] Y.T. Wang, Y.X. Zhou, J. Sokolov, B. Rigas, K. Levon, M. Rafailovich, A potentiometric protein sensor built with surface molecular imprinting method, Biosens. Bioelectron. 24 (2008) 162-166.

[26] X. Zhang, A. Tretjakov, M. Hovestaedt, G. Sun, V. Syritski, J. Reut, R. Volkmer, K. Hinrichs, J. Rappich, Electrochemical functionalization of gold and silicon surfaces by a maleimide group as a biosensor for immunological application, Acta Biomater. 9 (2013) 5838-5844

[27] A. Düzgün, A. Maroto, T. Mairal, C. O’Sullivan, F.X. Rius, Solid-contact potentiometric aptasensor based on aptamer functionalized carbon nanotubes for the direct determination of proteins, Analyst 135 (2010) 1037-1041

[28] A.P. Washe, S. Macho, G.A. Crespo, F.X. Rius, Potentiometric online detection of aromatic hydrocarbons in aqueous phase using carbon nanotube-based sensors, Anal. Chem. 82 (2010) 8106-8112.

\section{Biographies}

Enguang Lv is pursuing a doctor's degree at Yantai Institute of Coastal Zone Research, Chinese Academy of Sciences. His research interest is electrochemical biosensors.

Jiawang Ding is currently working as an associate professor at Yantai Institute of Coastal Zone Research, Chinese Academy of Sciences. From 2016 to 2017, he did his joint research at Åbo Akademi University (Finland) and University of Geneva (Switzerland). His research interests include electrochemical sensors and biosensors.

Wei Qin received his doctor's degree from Nanjing University in 1998. From 1999 to 2003, he did his postdoctoral research at Swiss Federal Institute of Technology (ETH), University of South Carolina and University of Michigan, respectively. He has been working as a professor at Yantai Institute of Coastal Zone Research, Chinese Academy of Sciences since 2006. His research interests include chemical sensors and biosensors for environmental analysis. 